\title{
ON THE MAXIMUM PRINCIPLE FOR PRINCIPAL CURVATURES
}

\author{
NINA M. IVOCHKINA \\ St.-Petersburg State University of Architecture and Civil Engineering \\ 2-Krasnoarmeiskaya, 4, 198005 St.-Petersburg, Russia \\ E-mail: rector@spice.spb.su
}

\begin{abstract}
The paper contains the estimates from above of the principal curvatures of the solution to some curvature equations. A correction of the author's previous argument is presented.
\end{abstract}

1. Introduction. The natural domain of definition of curvature operators is the set of $C^{2}$-smooth $n$-dimensional surfaces imbedded in $\mathbb{R}^{n+1}, n \geq 2$. Indeed, we assign to any $\Gamma \subset \mathbb{R}^{n+1}$ the vector-operator $k[\Gamma]=\left(k^{1}, \ldots, k^{n}\right)[\Gamma]$ whose components are the principal curvatures of $\Gamma$. Then a curvature operator $F$ takes the form

$$
F[\Gamma]=f(k[\Gamma])=f(k)[\Gamma]
$$

with some known function $f$ defined in $\mathbb{R}^{n}$. In order to circumvent the problem of global numbering of the principal curvatures over $\Gamma$ we always assume $f$ to be a symmetric function in $\mathbb{R}^{n}$.

Denote by $D(F)$ the subset of surfaces satisfying

$$
\begin{gathered}
\frac{\partial f}{\partial k^{i}}[\Gamma]>0, \quad i=1, \ldots, n, \\
\sum_{i, j=1}^{n} \frac{\partial^{2} f}{\partial k^{i} \partial k^{j}}[\Gamma] \xi^{i} \xi^{j} \leq 0, \quad|\xi|=1 .
\end{gathered}
$$

We assume here $D(F) \neq \emptyset$.

The simplest examples of curvature operators are the operators of $m$-curvature:

$$
\mu_{m}[\Gamma]=\frac{S_{m}(k[\Gamma])}{\left(\begin{array}{c}
n \\
m
\end{array}\right)}, \quad m=1, \ldots, n,
$$

where $S_{m}$ is the $m$ th elementary symmetric function. The set $D\left(\mu_{m}\right)$ admits the following

1991 Mathematics Subject Classification: 35J60, 53A99.

The paper is in final form and no version of it will be published elsewhere. 
description [4]:

$$
D\left(\mu_{m}\right)=\left\{\Gamma \subset \mathbb{R}^{n+1}: \mu_{i}[\Gamma]>0, i=1, \ldots, m\right\} .
$$

In the case of complete bounded surfaces it suffices to require only $\mu_{m}[\Gamma]>0$. The set $D\left(\mu_{n}\right)$ consists of all convex surfaces. We use the term " $m$-convex surface" introduced in [10] for surfaces from $D\left(\mu_{m}\right), m=1, \ldots, n$.

Any $n$-dimensional surface smoothly imbedded in $\mathbb{R}^{n+1}$ may be interpreted locally as the graph of some smooth function $u$, i.e.

$$
\Gamma_{l o c}=\left\{x \in \mathbb{R}^{n}:|x|<\varepsilon, x^{n+1}=u(x)\right\} .
$$

Then the operator (1) can be viewed as a second-order differential operator on $C^{2}\left(B_{\varepsilon}\right)$, $B_{\varepsilon} \subset \mathbb{R}^{n}$. We then write

$$
F[u]=f(k[u])=f(k)[u],
$$

where the components of $k[u]$ are the eigenvalues of the following extremal problem:

$$
\begin{gathered}
u_{x x} \tau_{i}=w k^{i} G \tau_{i}, \quad i=1, \ldots, n, \\
u_{x x}=\left(u_{i j}\right), \quad u_{i j}=\frac{\partial^{2} u}{\partial x^{i} \partial x^{j}}, \quad w=\sqrt{1+u_{x}^{2}}, \quad G=\delta_{i j}+u_{i} u_{j} .
\end{gathered}
$$

The symmetry of $f$ permits us to rewrite the operator (4) in some neighbourhood of a fixed point $x_{0}$ as

$$
f[u]=F\left(w u_{(x x)}\right),
$$

where $u_{(x x)}=\left(u_{(i j)}\right)$,

$$
v_{\left(i_{1} \ldots i_{p}\right)}=\frac{\partial^{p} v}{\partial x^{j_{1}} \ldots \partial x^{j_{p}}} \tau_{i_{1}}^{j_{1}} \ldots \tau_{i_{p}}^{j_{p}}=v_{j_{1} \ldots j_{p}} \tau_{i_{1}}^{j_{1}} \ldots \tau_{i_{p}}^{j_{p}}
$$

and $\left\{\tau_{i}: i=1, \ldots, n\right\}$ is any system of vector fields satisfying

$$
\left(G \tau_{i}, \tau_{j}\right)=\delta_{i j} .
$$

From now on the summation over repeated indices is assumed unless otherwise stated. In notations (6)-(8), inequalities (2), (3) become

$$
\begin{gathered}
\frac{\partial F\left(u_{(x x)} w\right)}{\partial u_{(i j)}} \xi^{i} \xi^{j}>0, \quad|\xi|=1, \\
\frac{\partial^{2} F}{\partial u_{(i j)} \partial u_{(k l)}} \xi^{i j} \xi^{k l} \leq 0, \quad \xi^{i j}=\xi^{j i} .
\end{gathered}
$$

If we do not want to use explicitly the vector fields (8), we have to express the operator (4) in the general form

$$
F[u]=F\left(u_{x}, u_{x x}\right),
$$

assuming the invariance of the function $F$ under rotations of the vector $X=(x, u)$ in $\mathbb{R}^{n+1}$. To give a sample of representation (11) we write out the operators $\mu_{m}$ :

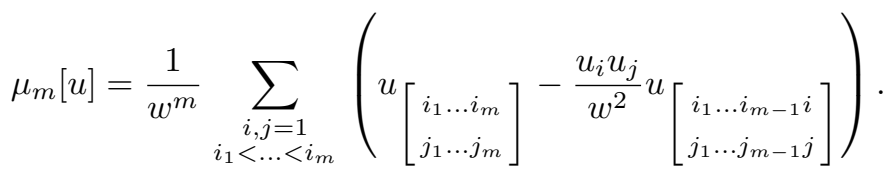


Here

$$
u\left[\begin{array}{l}
i_{1} \ldots i_{m} \\
j_{1} \ldots j_{m}
\end{array}\right]
$$

is the $m$-minor of $u_{(x x)}$ with rows and columns numbered by $i_{1}<\ldots<i_{m}$ and $j_{1}<$ $\ldots<j_{m}$. Conditions (2), (3) transformed to the form (11) look like (9), (10) with indices without brackets.

The curvature equations contain by definition the curvature operator and read

$$
F[u]=g[u],
$$

where $g$ is a differential operator of order at most one. In view of (11) equation (12) may be considered as a fully nonlinear differential equation of second order. On the set $D(F)$ it becomes of elliptic type.

The program of investigation of curvature equations follows one for equations containing the eigenvalues of the Hesse matrix instead of curvatures $[1,4,5]$. However, the analysis of curvature equations requires far more complicated techniques $[2,3,6,7,9]$. Two different approaches were developed to the problem of calculations in the curvature case. The authors of $[2,3,9]$ exploited the rotation invariance of the operator (11) and chose a suitable cartesian coordinate system. Then equation (12) was directly differentiated in that system. In [6] all the difficulties were accumulated when differentiating the principal curvatures in some local cartesian system. However, this procedure is incorrect in general, which was pointed out to the author by Prof. N. S. Trudinger during a Session of Banach Semester, Warsaw, October 93.

The present paper contains a correction to the relevant reasoning in [6] as well as some new results of geometrical nature. Samples of them are:

Proposition 1. Let a convex $C^{4}$-surface $\Gamma$ be of constant Gauss curvature. Then the maximal value of its principal curvatures is attained at the boundary $\partial \Gamma$.

Proposition 1 seems to be known in differential geometry, though the author failed to find references. Anyway, the fact that a complete convex surface of constant Gauss curvature has to be a sphere, a fact following from Proposition 1, is well known.

The case of Gauss curvature corresponds to $\mu_{n}$ (see (4)) and the assertion of Proposition 1 cannot be extended to other $\mu_{m}$. However, some analogue is valid for any $m$ for surfaces $\Gamma$ which are graphs over some domain $\Omega \subset \mathbb{R}^{n}$. Denote $\widetilde{k}^{i}=w k^{i}, i=1, \ldots, n$ $($ see $(5))$.

Proposition 2. Let an $m$-convex $C^{4}$-surface $\Gamma$ be of constant $m$-curvature. Then the function $\widetilde{k}_{0}=\max _{i} \widetilde{k}^{i}$ attains its maximal value over $\Omega$ at the boundary $\partial \Omega$.

2. Some preliminary notes. One of the objectives when analyzing curvature equations is to bound from above the values of the principal curvatures of an unknown surface, that is, to bound from above the values of the function

$$
k_{0}(X)=\max _{i=1, \ldots, n} k^{i}(X), \quad X \in \Gamma .
$$


The continuous function (13) attains its maximum at some point $X_{0} \in \Gamma$. We now consider the case $X_{0} \in \Gamma \backslash \partial \Gamma$. By inequality (2) the application of the classical maximum principle for second order elliptic differential equations looks reasonable. The technical problem we are facing is to find an auxiliary function $W$ such that

(i) $W$ is at least $C^{4}$-smooth in some neighbourhood of $X_{0}$;

(ii) $W$ attains its maximum at $X_{0}=\left(x_{0}, u\left(x_{0}\right)\right)$ and

$$
k_{0}\left(X_{0}\right) \leq c W\left(x_{0}\right),
$$

where $c$ is some constant under control;

(iii) there exists a positive matrix $A=\left(A^{i j}\right)$ such that in some local cartesian system the inequality

holds if $W\left(x_{0}\right) \gg 1$.

$$
\left(A^{i j} W_{i j}\right)\left(x_{0}\right)>0
$$

The cartesian system involved in the definition of $W$ depends on $X_{0}$. We call it a primary system if the matrix $\left(G^{1 / 2} u_{x x} G^{1 / 2}\right)\left(x_{0}\right)$ is diagonal.

The function $W$ will be composed of functions $u_{(i j)}=u_{k l} \tau_{i}^{k} \tau_{j}^{l}, i=1, \ldots, n$. The vectors $\tau_{i}, i=1, \ldots, n$, are always supposed to satisfy (8). This type of auxiliary function was introduced in [8], devoted to the Monge-Ampère equation. As an advantage of calculating in the primary system we always have

$$
u_{(i i)}\left(x_{0}\right)=w k^{i}\left(X_{0}\right), \quad i=1, \ldots, n .
$$

We emphasize that relations (14) fail in general in the neighbourhood of $x_{0}$.

Let $\left\{\eta^{i}\right\}$ be the dual system to $\left\{\tau_{i}\right\}$, i.e.

$$
\eta_{k}^{i} \tau_{j}^{k}=\delta_{j}^{i}, \quad \eta_{i}^{k} \tau_{k}^{j}=\delta_{i}^{j}
$$

The following relations are helpful:

$$
\begin{aligned}
& \left(\eta_{i}, \eta_{j}\right)=(G)_{i j}=\delta_{i j}+u_{i} u_{j}, \\
& \left(\tau^{i}, \tau^{j}\right)=\left(G^{-1}\right)^{i j}=\delta^{i j}-\frac{u_{i} u_{j}}{w^{2}} .
\end{aligned}
$$

The simplest choice of $\tau_{i}, \eta^{i}, i=1, \ldots, n$, is $\tau_{i}=\hat{\tau}_{i}, \eta^{i}=\hat{\eta}^{i}$, where

$$
\begin{aligned}
& \hat{\tau}_{i}^{k}=\left(G^{-1 / 2}\right)_{i}^{k}=\delta_{i}^{k}-\frac{u_{k} u_{i}}{w(1+w)}, \\
& \hat{\eta}_{i}^{k}=\left(G^{1 / 2}\right)_{i}^{k}=\delta_{i}^{k}-\frac{u_{k} u_{i}}{1+w} .
\end{aligned}
$$

Since we are to differentiate $u_{(i i)}$ it is convenient to introduce

$$
c_{j k}^{i}=\eta_{l}^{i}\left(\tau_{j}^{l}\right)_{(k)}=\left(G \tau_{i},\left(\tau_{j}\right)_{(k)}\right) .
$$

For any vector fields (15)-(17), in the neighbourhood of $x_{0}$ the following identities are true:

$$
\begin{gathered}
c_{j, k}^{i}+c_{i, k}^{j}=-\left(G_{(k)} \tau_{i}, \tau_{j}\right), \\
u_{(i j),(k)}=u_{(i j k)}+u_{(i p)} c_{(j k)}^{p}+u_{(j p)} c_{i, k}^{p}, \quad i, j, k=1, \ldots, n .
\end{gathered}
$$

Formula (22) holds since

$$
u_{p r}=u_{(i j)} \eta_{p}^{i} \eta_{r}^{j}, \quad p, r=1, \ldots, n
$$


At $x_{0}$ relation $(22)$ turns out to be equivalent to

$$
u_{(i j),(k)}=u_{(i j k)}+\left(u_{(j j)}-u_{(i i)}\right) c_{i k}^{j}-u_{(i i)}\left(G_{(k)} \tau_{i}, \tau_{j}\right) .
$$

Formula (24) reveals the peculiarity of the case $u_{(i i)}\left(x_{0}\right)=u_{(j j)}\left(x_{0}\right)$.

Finally, we write out $c_{j, k}^{i}$ explicitly for the vector fields (18), (19):

$$
\hat{c}_{j k}^{i}=-\frac{u_{(i k)} u_{(j)}+u_{(j k)} u_{i}}{1+w} .
$$

Note that the relations of this section are true for sufficiently smooth vectors $\left\{\tau_{i}\right\}$. Further on we keep at least $C^{2}$-smoothness for any choice of $\left\{\tau_{i}\right\}$.

3. The equation of prescribed Gauss curvature $\left(^{1}\right)$. We consider the set of $n$-dimensional $C^{4}$-surfaces imbedded in $\mathbb{R}^{n+1}$ and a function $h \in C^{2}\left(\mathbb{R}^{n+1}\right)$, and rewrite equation (4) with $m=n$ in geometrical terms as follows:

$$
\mu_{n}[\Gamma](X)=h^{n}(X)
$$

Here $h^{n}$ acquires the sense of a prescribed Gauss curvature of $\Gamma$. The set $D\left(\mu_{n}\right)$ consists of all strictly convex $C^{4}$-surfaces.

We choose some point $X_{0} \in \Gamma$ and a cartesian coordinate system about it requiring the vector $(0, \ldots, 1)$ to be normal to $\Gamma$. There exists a neighbourhood $B_{\varepsilon}\left(X_{0}\right)$ such that

$$
\Gamma \cap B_{\varepsilon}\left(X_{0}\right)=\left\{|x|<\varepsilon, x^{n+1}=u(x)\right\},
$$

where the function $u$ is as smooth as $\Gamma$. We also require this cartesian system to be primary at $x_{0}$. For $\left\{\tau_{i}\right\}$ we take the system (18) and rewrite equation (26) as

$$
\operatorname{det}^{1 / n}\left(\frac{u_{(x x)}}{w}\right)=h(x, u) \text {. }
$$

It follows from (5) that the principal curvatures of $\Gamma$ are the eigenvalues of the matrix $u_{(x x)} / w$ in the neighbourhood of $x_{0}$. From the choice of the cartesian system, at $x_{0}$ we have

$$
u_{x x}=u_{(x x)}=\left(\delta_{j}^{i} k^{j}\right) .
$$

The crucial point of the further reasoning is the formula

$$
\begin{aligned}
& \left(\left(\frac{u_{(i i)}}{w}\right)_{(j j)}-\left(\frac{u_{(j j)}}{w}\right)_{(i i)}\right)\left(x_{0}\right) \\
& =\left(u_{(j j)}^{2} u_{(i i)}-u_{(i i)}^{2} u_{(j j)}\right)\left(x_{0}\right)=\left(k^{j} k^{i}\left(k^{j}-k^{i}\right)\right)[\Gamma]\left(X_{0}\right) .
\end{aligned}
$$

This follows from $u_{x}\left(x_{0}\right)=0$, which leads to $\hat{c}_{j k}^{i}\left(x_{0}\right)=0$ (see $(20)$ ).

TheOREM 3. Let $\Gamma$ be a convex $C^{4}$-solution to equation (26). Then either $k_{0}[\Gamma]$ attains its maximum at the boundary $\partial \Gamma$ or

$$
k_{0}[\Gamma]<2 \max _{B_{R}}\left(\sqrt{\frac{\Lambda^{-}\left(h_{X X}\right)+\left|h_{X}\right|^{2}}{h}} \cdot \frac{h(X)}{2^{1 / n}}\right),
$$

$\left({ }^{1}\right)$ The content of Section 3 was worked out with F. Tomi during the author's stay at Heidelberg University, February, 1993. 
where $B_{R}$ is a ball containing $\Gamma, \Lambda\left(h_{X X}\right)$ is the minimal negative eigenvalue of $h_{X X}$ and zero if $h_{X X} \geq 0$, and $\Lambda^{-}=|\Lambda|$.

Proof. Let $X_{0}$ be a point where the maximal value of $k_{0}$ is attained (see (13)). Denote

$$
u_{\langle x x\rangle}=\left(u_{\langle i j\rangle}\right), \quad u_{\langle i\rangle}=\frac{u_{(i)}}{\sqrt{w}},
$$

where the indices correspond to the above described cartesian system and the vector fields (18). By (28),

$$
k_{0}\left(X_{0}\right)=\max _{i=1, \ldots, n} \max _{|x|<\varepsilon} u_{\langle i i\rangle}(x) .
$$

Denote by $\gamma$ the index which realizes the maximum (31), i.e.

$$
u_{\langle\gamma \gamma\rangle}\left(x_{0}\right)=k_{0}\left(X_{0}\right) .
$$

Then

$$
\left(\sum_{i=1}^{n} \frac{\partial F\left(u_{\langle x x\rangle}\right)}{\partial u_{\langle i i\rangle}} u_{\langle\gamma \gamma\rangle,\langle i i\rangle}\right)\left(x_{0}\right) \leq 0
$$

with $F\left(u_{\langle x x\rangle}\right)=\operatorname{det}^{1 / n} u_{\langle x x\rangle}$.

On the other hand, differentiating twice equation (28) we get

$$
\sum_{i, j=1}^{n} \frac{\partial F\left(u_{\langle x x\rangle}\right)}{\partial u_{\langle i i\rangle}} u_{\langle i i\rangle,\langle\gamma \gamma\rangle}=h_{\langle\gamma \gamma\rangle}-\sum_{i, j, k, l=1}^{n} \frac{\partial^{2} F\left(u_{\langle x x\rangle}\right)}{\partial u_{\langle i j\rangle} \partial u_{\langle k l\rangle}} u_{\langle i j\rangle,\langle\gamma\rangle} u_{\langle k l\rangle,\langle\gamma\rangle}
$$

in the neighbourhood of $x_{0}$. By the properties of the primary coordinate system and concavity of $F$ over positive matrices, relation (32), when combined with (33) and (29), yields

$$
\left(\frac{\partial F\left(u_{\langle x x\rangle}\right)}{\partial u_{\langle i i\rangle}} k^{i} k_{0}\left(k_{0}-k^{i}\right)\right)\left(x_{0}\right)-\Lambda^{-}\left(h_{X X}\right)+\left|h_{X}\right| k_{0}\left(X_{0}\right) \leq 0 .
$$

Since

$$
\left(\frac{\partial F\left(u_{\langle x x\rangle}\right)}{\partial u_{\langle i i\rangle}} k^{i}\right)\left(x_{0}\right)=\frac{1}{n} h\left(X_{0}\right), \quad i=1, \ldots, n,
$$

we are facing the alternative: either $k^{i}\left(X_{0}\right) \leq \frac{1}{2} k_{0}\left(X_{0}\right)$ at least for one index $i$, which implies

$$
k_{0}[\Gamma]<2 \max _{B_{R}} \sqrt{\frac{\Lambda^{-}\left(h_{X X}\right)+\left|h_{X}\right|^{2}}{h}},
$$

or $k^{i}\left(X_{0}\right) \geq \frac{1}{2} k_{0}\left(X_{0}\right)$, and then

$$
k_{0}[\Gamma]<2^{(n-1) / n} \max _{B_{R}} h(X) .
$$

In both the cases estimate (30) is valid.

For those who do not like vector fields we write out the function $W$, which was the subject of estimations in Theorem 3, without any conventions in the primary system:

$$
W=\frac{1}{w} u_{i j}\left(\delta_{\gamma}^{i}-\frac{u_{i} u_{j}}{w(1+w)}\right)\left(\delta_{j}^{i}-\frac{u_{j} u_{j}}{w(1+w)}\right) .
$$


Inequalities (32)-(34) in terms of $W$ may be summarized as follows:

$$
\begin{aligned}
0 & \geq\left(\sum_{i, j=1}^{n} \frac{\partial F\left(u_{x}, u_{x x}\right)}{\partial u_{i j}} W_{i j}\right)\left(x_{0}\right) \\
& \geq\left(\sum_{i} \frac{\partial F\left(u_{x}, u_{x x}\right)}{\partial u_{i i}} u_{i i} W\left(W-u_{i i}\right)-\Lambda^{-}\left(h_{X X}\right)+\left|h_{X}\right| W\right)\left(x_{0}\right),
\end{aligned}
$$

where $F\left(u_{x}, u_{x x}\right)=\left(\operatorname{det} u_{x x} / w^{n+2}\right)^{1 / n}$.

Proposition 1 is close to being Theorem 3 with constant $h$ but more subtle. In order to prove the pure maximum principle stated in Proposition 1 we note that in the case of constant $h$ relation (34) becomes

$$
\sum_{i=1}^{n}\left(k_{0}-k^{i}\right)[\Gamma]\left(X_{0}\right) \leq 0 .
$$

The latter is only possible for $k^{i}\left(X_{0}\right)=k_{0}\left(X_{0}\right)$, which implies our surface is part of the sphere. Then $k_{0}[\Gamma]=$ const and certainly it attains its maximum at the boundary.

The analysis of relations $(29),(33),(34)$ shows that the presented sample of reasoning admits some widening of the class of curvature operators, provided we keep the convexity of $\Gamma$. On the other hand, this approach fails for non-convex surfaces, because it is impossible to control the sign of the commutator (29).

4. The description of auxiliary vector fields in the general case. It is easy to check that the assumption $w\left(x_{0}\right)=0$ leads in the primary system to the identities

$$
J_{i j}=\left(u_{(i i),(j j)}-u_{(j j),(i i)}\right)\left(x_{0}\right)=0, \quad i, j=1, \ldots, n,
$$

and it looks natural to try the maximum principle for $W=u_{(\gamma \gamma)}$. However, an attempt to fix $w\left(x_{0}\right)=1$ presupposes the rotation invariance of the problem in $\mathbb{R}^{n+1}$, which does not hold. Indeed, equation (12) in the neighbourhood of some $X_{0} \in \Gamma$ takes the form

$$
F\left(u_{(x x)} / w\right)=h \text {. }
$$

The latter is invariant under rotations of $\mathbb{R}^{n}$ only. Moreover, the points which supply the maximum value to $k_{0}[\Gamma]$ and $u_{(\gamma \gamma)}$ may be different because of the factor $1 / w$.

We have to omit the requirement $w\left(x_{0}\right)=1$. Therefore the invariance of the curvature operators in $\mathbb{R}^{n}$ is only available, and here the condition for $\Gamma$ to be the graph of some function $u$ in $\Omega \subset \mathbb{R}^{n}$ enters. We also require $F$ to be homogeneous, say of the first order, and rewrite equation (35) in the form

$$
F\left(u_{(x x)}\right)=w h, \quad x \in \Omega .
$$

In order to choose vector fields (15)-(17) properly we point out the degrees of freedom. Represent the matrix $G$ in the form

$$
G=C^{\prime} C, \quad C=B G^{1 / 2}, \quad B^{T}=B^{-1} .
$$

Any vector field defined by the formulae

$$
\eta_{j}^{i}=(C)_{j}^{i}, \quad \tau_{j}^{i}=\left(C^{-1}\right)_{j}^{i}, \quad i, j=1, \ldots, n,
$$


satisfies (15)-(17) in $\Omega$, and equation (12) has the form (36) for every cartesian system in $\mathbb{R}^{n}$. Consider the primary system about $x_{0} \in \Omega$ and assume

$$
B\left(x_{0}\right)=I \text {. }
$$

The freedom in constructing vector fields (38) is in the choice of $B(x)$ while keeping (39). Our aim is to obtain if possible the equalities

$$
u_{(i j),(k)}\left(x_{0}\right)=0, \quad i \neq j .
$$

Without loss of generality suppose $x_{0}=0$ and introduce numbers $\beta_{j, k}^{i}$ by the formulas

$$
\beta_{j k}^{i}=\hat{\beta}_{j p}^{i} \hat{\eta}_{k}^{p}(0), \quad i, j, k=1, \ldots, n,
$$

where

$$
\hat{\beta}_{i p}^{j}=\left(\frac{u_{(i j p)}+u_{(i i)} \hat{c}_{j p}^{i}+u_{(j j)} \hat{c}_{i p}^{j}}{u_{(i i)}-u_{(j j)}}\right)(0)
$$

when $u_{(i i)}(0) \neq u_{(j j)}(0)$, and

$$
\hat{\beta}_{j p}^{i}=0
$$

otherwise.

The numbers (41) are antisymmetric in $i, j$ and therefore the matrix

$$
B=\left(b_{j}^{i}\right)=\exp \left(\beta_{j k}^{i} x^{k}\right)
$$

is orthogonal.

LEMma 4. Assume the vector fields (38) are constructed on the base of (44), i.e.

$$
\eta_{j}^{i}=b_{p}^{i} \hat{\eta}_{j}^{p}, \quad \tau_{j}^{i}=\hat{\tau}_{p}^{i} b_{p}^{j} .
$$

Then relations $(40)$ hold if $u_{(i i)}\left(x_{0}\right) \neq u_{(j j)}\left(x_{0}\right)$, and

$$
c_{j k}^{i}\left(x_{0}\right)=\hat{c}_{j k}^{i}\left(x_{0}\right)
$$

otherwise.

Pro of. Write out (20) at $x_{0}$ for the vector fields (45):

$$
c_{j k}^{i}=\hat{c}_{j k}^{i}+\hat{\beta}_{i k}^{j} \text {. }
$$

Relation $(22)$ at $x_{0}$ reads

$$
u_{(i j),(k)}=u_{(i j k)}+u_{(i i)} \hat{c}_{j k}^{i}+u_{(j j)} \hat{c}_{i, k}^{j}+\hat{\beta}_{i k}^{j} u_{(i i)}+\hat{\beta}_{j k}^{i} u_{(j j)} .
$$

By antisymmetry and the choice (42) of $\beta_{j k}^{i}$ the latter is equivalent to (40) if $u_{(i i)}\left(x_{0}\right) \neq$ $u_{(j j)}\left(x_{0}\right)$. In the other case (47), (43) obviously lead to (46).

5. Some geometrical relations. The principal concern of this section is to handle the commutators $J_{i j}, i, j=1, \ldots, n$ (see the first lines of Section 4). To begin with we represent the derivative $u_{(i i),(k k)}$ in a special form.

LEMma 5. For any vector fields (14)-(17) the following formulas hold at $x_{0}$ :

$$
u_{(i i),(k k)}=u_{(i i k k)}+4 c_{i k}^{j} u_{(i j),(k)}+2\left(u_{(i i)}-u_{(j j)}\right)\left(c_{i, k}^{j}\right)^{2}-u_{(i i)}\left(G_{(k k)} \tau_{i}, \tau_{i}\right),
$$

$i, k=1, \ldots, n$, where notation (7) is used. 
P r o of. Formulas (48) result from the following calculations:

$$
\begin{gathered}
u_{(i i),(k k)}\left(x_{0}\right)=\left(u_{(i i k k)}+4 u_{(i j k)} c_{i k}^{j}+2 u_{(j j)}\left(c_{i k}^{j}\right)^{2}+2 u_{(i i)} \eta_{p}^{i}\left(\tau_{i}^{p}\right)_{(k k)}\right)\left(x_{0}\right), i=1, \ldots, n, \\
u_{(i j k)}\left(x_{0}\right)=\left(u_{(i j),(k)}+\left(u_{(i i)}-u_{(j j)}\right) c_{i k}^{j}+u_{(i i)}\left(G_{(k)} \tau_{i}, \tau_{j}\right)\right)\left(x_{0}\right), \\
\eta_{p}^{i}\left(\tau_{i}^{p}\right)_{(k k)}=\left(G \tau_{i},\left(\tau_{i}\right)_{(k k)}\right)=-\frac{1}{2}\left(\left(G_{(k k)} \tau_{i}, \tau_{i}\right)+2\left(G\left(\tau_{i}\right)_{(k)},\left(\tau_{i}\right)_{(k)}\right)\right. \\
\left.+4\left(G_{(k)} \tau_{i},\left(\tau_{i}\right)_{(k)}\right)\right), \quad i=1, \ldots, n, \\
\left(G\left(\tau_{i}\right)_{(k)},\left(\tau_{i}\right)_{(k)}\right)=\sum_{j=1}^{n}\left(c_{i k}^{j}\right)^{2}, \quad\left(G_{(k)} \tau_{i},\left(\tau_{i}\right)_{(k)}\right)=\left(G_{(k)} \tau_{i}, \tau_{j}\right) c_{i k}^{j} .
\end{gathered}
$$

We further consider the term $c_{i k}^{j} u_{(i j),(k)}$ in (48). In the author's paper [6] the vector fields $\left\{\tau_{i}: i=1, \ldots, n\right\}$ were supposed to be the eigenvectors of the matrix $G^{-1 / 2} u_{x x} G^{-1 / 2}$ in the whole neighbourhood of $x_{0}$. This construction implied the identities $u_{(i j),(k)}=0$, $i, j=1, \ldots, n$. However, such choice of vector fields is only possible when all principal curvatures are different at $X_{0}$. The correction presented below covers the case of equal curvatures.

We introduce the following ordering: $u_{(11)}\left(x_{0}\right)<\ldots<u_{(\gamma \gamma)}\left(x_{0}\right), \gamma \leq n$. Then $u_{(i i)}\left(x_{0}\right) \leq u_{(\gamma \gamma)}\left(x_{0}\right), i=1, \ldots, n$. Split $\{1, \ldots, n\}$ into subsets $I_{p}, p=1, \ldots, \gamma$, where $I_{p}=\left\{1 \leq i \leq n: u_{(i i)}\left(x_{0}\right)=u_{(p p)}\left(x_{0}\right)\right\}$.

Lemma 6. For any nonnegative numbers $a^{p}, p=1, \ldots, \gamma$, and $r, 1 \leq r \leq \gamma$, the following relation holds at $x_{0}$ :

$$
\begin{aligned}
\sum_{p=1}^{\gamma} a_{p} \sum_{k \in I_{r}} \sum_{i, j \in I_{p}} c_{i k}^{j} u_{(i j),(k)} & =a_{r} \sum_{i, j, k \in I_{r}} c_{i k}^{j} u_{(i j),(k)} \\
& =\sum_{p=1}^{\gamma} a_{p} \sum_{i \in I_{p}} \sum_{k, j \in I_{r}} c_{k i}^{j} u_{(k j),(i)} .
\end{aligned}
$$

Proof. By (46) (see (25))

$$
c_{j k}^{i}\left(x_{0}\right)=\left(\frac{\delta_{k}^{i} u_{(j)}+\delta_{k}^{i} u_{i}}{1+w} u_{(k k)}\right)\left(x_{0}\right), \quad i, j \in I_{p}, p=1, \ldots, \gamma
$$

These equalities mean that actually the summation on both sides of (49) is over $i, j, k \in I_{r}$, $p=r$.

Lemma 7. Let $a^{i}, i=1, \ldots, n$, be nonnegative. Then for any vector fields (14)-(17), $k \in I_{\gamma}$, the following equality holds at $x_{0}$ :

$$
\text { (50) } \sum_{u_{(i i)} \geq u_{(j j)}} a^{i}\left(u_{(i i)}-u_{(j j)}\right)\left(\left(c_{i k}^{j}\right)^{2}-\left(c_{j k}^{i}\right)^{2}\right)=a^{k} \sum_{j=1}^{n}\left(u_{(k k)}-u_{(j j)}\right)\left(\left(c_{k k}^{j}\right)^{2}-\left(c_{j k}^{k}\right)^{2}\right) \text {. }
$$

Proof. By the summation condition in (50), formulas (21) at $x_{0}$ become

$$
c_{j k}^{i}+c_{i k}^{j} \equiv u_{(i)} u_{(j k)}+u_{(j)} u_{(i k)}=\delta_{k}^{i} u_{(j)} u_{(k k)} .
$$

Therefore all the nonvanishing terms on the left-hand side of (50) are those on its righthand side. 
Lemma 8. Assume that nonnegative $a^{i}, i=1, \ldots, n$, satisfy $a^{i} \geq a^{j}$ if $i \leq j$. Then for vector fields (38), (44) related to $x_{0}$ the following inequality holds at $x_{0}$ :

$$
\sum_{k \in I_{\gamma}} a^{i}\left[u_{(k k),(i i)}\right] \geq \sum_{k \in I_{\gamma}} a^{i}\left[u_{(i i)}\left(G_{(k k)} \tau_{i}, \tau_{i}\right)\right],
$$

where $[\Phi(i, j)]=\Phi(i, j)-\Phi(j, i)$.

Proof. By (40), (48), (49),

$$
\begin{aligned}
& \sum_{k \in I_{\gamma}} a^{i}\left[u_{(k k),(i i)}\right] \\
& =\sum_{k \in I_{\gamma}} \sum_{i, j=1}^{n} a^{i}\left(\left(u_{(k k)}-u_{(j j)}\right)\left(c_{k i}^{j}\right)^{2}-\left(u_{(i i)}-u_{(j j)}\right)\left(c_{i k}^{j}\right)^{2}+\left[u_{(i i)}\left(G_{(k k)} \tau_{i}, \tau_{i}\right)\right]\right) .
\end{aligned}
$$

We use the monotonicity of $a^{i}$ and Lemma 7 to get

$$
\begin{aligned}
\sum_{i, j=1}^{n} a^{i}\left(u_{(i i)}\right. & \left.-u_{(j j)}\right)\left(c_{i, k}^{j}\right)^{2} \\
& =\sum_{u_{(i i)}>u_{(j j)}} a^{i}\left(u_{(i i)}-u_{(j j)}\right)\left(c_{i k}^{j}\right)^{2}+\sum_{u_{(i i)}<u_{(j j)}} a^{i}\left(u_{(i i)}-u_{(j j)}\right)\left(c_{i k}^{j}\right)^{2} \\
& =\sum_{u_{(i i)}>u_{(j j)}}\left(a^{i}\left(u_{(i i)}-u_{(j j)}\right)\left(c_{i k}^{j}\right)^{2}-a^{j}\left(u_{(j j)}-u_{(i i)}\right)\left(c_{j k}^{i}\right)^{2}\right) \\
& \leq \sum_{u_{(i i)}>u_{(j j)}} a^{i}\left(u_{(i i)}-u_{(j j)}\right)\left(\left(c_{i k}^{j}\right)^{2}-\left(c_{j k}^{i}\right)^{2}\right) \\
& =a^{k} \sum_{j=1}^{n}\left(u_{(k k)}-u_{(j j)}\right)\left(\left(c_{k k}^{j}\right)^{2}-\left(c_{j k}^{k}\right)^{2}\right) .
\end{aligned}
$$

Combining the latter with (52) we obtain (51).

Note that we never use in this section any special properties of $\Gamma$ or $u$ but smoothness.

6. Application to the general curvature equations. We now return to the curvature equations in the form (36) which presupposes the graphs over $\Omega$ to be the only surfaces of interest. We shall make use as well of the form (4) when exploiting the symmetry of the considered functions $f$. We state a helpful and well-known (see [1]) consequence of symmetry and concavity of $f$.

Lemma 9. Let $f$ be symmetric and concave on some convex subset $B \subset \mathbb{R}^{n}$. Then $x^{i} \leq x^{j}$ implies $\partial f / \partial x^{i} \geq \partial f / \partial x^{j}$ for $x=\left(x^{1}, \ldots, x^{n}\right)$.

Lemma 9 follows from Taylor's formula. Indeed,

$$
\begin{aligned}
0= & f\left(x^{2}, x^{1}\right)-f\left(x^{1}, x^{2}\right) \\
= & \left(\frac{\partial f\left(x^{1}, x^{2}\right)}{\partial x^{1}}-\frac{\partial f\left(x^{1}, x^{2}\right)}{\partial x^{2}}\right)\left(x^{2}-x^{1}\right) \\
& +\int_{0}^{1}(1-\tau)\left(\frac{\partial^{2} f}{\left(\partial \widetilde{x}^{1}\right)^{2}}-2 \frac{\partial^{2} f}{\partial \widetilde{x}^{1} \partial \widetilde{x}^{2}}+\frac{\partial^{2} f}{\left(\partial \widetilde{x}^{2}\right)^{2}}\right) d \tau\left(x^{2}-x^{1}\right)^{2},
\end{aligned}
$$


where $\widetilde{x}^{1}=(1-\tau) x^{2}+\tau x^{1}, \widetilde{x}^{2}=(1-\tau) x^{1}+\tau x^{2}, \tau \in[0,1]$.

TheOREm 10. Assume the function $f$ in (4) to be homogeneous of the first order and symmetric. Assume also the image of $D(F)$ in $\mathbb{S}^{n}$, where $\mathbb{S}^{n}$ is the set of all symmetric matrices, to be convex. Then for any $C^{4}$-solution $u \in D(F)$ (viewed as a subset of $\left.C^{2}(\Omega)\right)$ to equation $(36)$ the following alternative holds: either $\widetilde{k}_{0}(x)=\left(w k_{0}\right)(x)$ attains its maximum at the boundary $\partial \Omega$ or

$$
\widetilde{k}_{0}^{2}(x) \leq \max _{\mathcal{B}_{R}} \frac{w^{2} l(\gamma)}{h}\left(2 \Lambda^{-}\left(h_{X X}\right)+9 \frac{l(\gamma)}{h} w^{2}\left|h_{X}\right|^{2}\right),
$$

where $\mathcal{B}_{R}=\{(x, u):|x|+|u|<R\}$.

Proof. Assume $\widetilde{k}_{0}(x)$ attains its maximum at $x_{0} \in \Omega$ and introduce the function

$$
W=\sum_{k \in I_{\gamma}} u_{(k k)}
$$

which is defined in some neighbourhood of $x_{0}$ where $u_{(k k)}=u_{i j} \tau_{k}^{i} \tau_{k}^{j}$ corresponds to (38), (44), and $I_{\gamma}$ is related to $x_{0}$ in the fashion described above. It is obvious that

$$
\widetilde{k}_{0}\left(x_{0}\right)=\frac{1}{l(\gamma)} W\left(x_{0}\right)=u_{(k k)}\left(x_{0}\right),
$$

where $l(\gamma)$ is the number of elements of $I_{\gamma}$. Moreover,

$$
W(x) \leq W\left(x_{0}\right), \quad|x|<\varepsilon,
$$

for some sufficiently small $\varepsilon$. Therefore,

$$
\begin{gathered}
W_{(i)}\left(x_{0}\right)=u_{(k k),(i)}\left(x_{0}\right)=0, \quad i=1, \ldots, n, k \in I_{\gamma}, \\
\sum_{i, j=1}^{n}\left(\frac{\partial F}{\partial u_{i j}} W_{i j}\right)\left(x_{0}\right) \equiv \sum_{i=1}^{n}\left(\frac{\partial F}{\partial u_{(i i)}} W_{(i i)}\right)\left(x_{0}\right) \equiv \sum_{k \in I_{\gamma}} a^{i} u_{(k k),(i i)} \leq 0 .
\end{gathered}
$$

We rewrite the latter expression as

$$
\sum_{k \in I_{\gamma}} a^{i} u_{(k k),(i)}=\sum_{k \in I_{\gamma}} a^{i}\left[u_{(k k),(i i)}\right]+\frac{\partial F}{\partial u_{(i i)}} u_{(i i),(k k)} .
$$

From (36) and the concavity of $F$ (see (10)), we have

$$
\sum_{i=1}^{n}\left(\frac{\partial F}{\partial u_{(i i)}} u_{(i i),(k k)}\right)\left(x_{0}\right) \geq(h w)_{(k k)}\left(x_{0}\right) .
$$

On the other hand, (36) combined with (54) and Lemma 8 lead to

$$
\sum_{k \in I_{\gamma}} a^{i}\left[u_{(k k),(i i)}\right]\left(x_{0}\right)=-2 u_{(k)} u_{(k k)}(h w)_{(k)} .
$$

Therefore (55) and (56) give rise to the inequality

$$
\sum_{k \in I_{\gamma}}\left((h w)_{(k k)}-2 u_{(k)} u_{(k k)}(h w)_{(k)}\right)\left(x_{0}\right) \leq 0 .
$$

The position of $w$ in (57) turns out to be convenient for our aims, since at $x=x_{0}$,

$$
w_{(k)}=w u_{(k)} u_{(k k)},
$$


Keeping in mind that

$$
w_{(k k)}=\frac{1}{w} u_{(k k)}^{2}+w u_{(p)} u_{(k k p)}, \quad u_{(k k p)}=u_{(k k),(p)}+2 \delta_{k}^{p} u_{(p)} u_{(k k)}^{2} .
$$

$$
W^{2}\left(x_{0}\right)=\frac{1}{l(\gamma)} \sum_{k \in I_{\gamma}} u_{(k k)}^{2}\left(x_{0}\right)
$$

we see that (57) implies the final inequality

$$
\frac{h}{w l(\gamma)} W^{2}\left(x_{0}\right) \leq w\left(\Lambda^{-}\left(h_{X X}\right)+3\left|h_{X}\right| W\right)\left(x_{0}\right) .
$$

The estimate (53) is a simple consequence of (58).

In contrast to Proposition 1 the assertion of Proposition 2 is a direct corollary of the estimate (53).

Acknowledgments. This work was supported by RU-grant 94-26-1068 and by an SF grant.

\section{References}

[1] L. Caffarelli, L. Nirenberg and J. Spruck, The Dirichlet problem for nonlinear second-order elliptic equations III. Functions of the eigenvalues of the Hessian, Acta Math. 155 (1985), 261-301.

[2] -, - , - Nonlinear second-order elliptic equations IV. Starshaped compact Weingarten hypersurfaces, in: Current Topics in Partial Differential Equations, Y. Olya, K. Kasahara and N. Shimajura (eds.), Kinokunize Co., Tokyo, 1986, 1-26.

[3] - , - - , Nonlinear second order elliptic equations $V$. The Dirichlet problem for Weingarten hypersurfaces, Comm. Pure Appl. Math. 41 (1988), 47-70.

[4] N. M. Ivochkina, A description of the stability cones generated by differential operators of Monge-Ampère type, Mat. Sb. 122 (1983), 265-275 (in Russian); English transl. in Math. USSR-Sb. 50 (1985).

[5] -, Solution of the Dirichlet problem for some equations of Monge-Ampère type, Mat. Sb. 128 (1985), 403-415 (in Russian); English transl. in Math. USSR-Sb. 56 (1987).

[6] -, Solution of the Dirichlet problem for the $m$-th order curvature equations, Mat. Sb. 180 (1989), 867-887 (in Russian); English transl. in Math. USSR-Sb. 67 (1990).

[7] -, The Dirichlet problem for $m$-th order curvature equations, Algebra i Analiz 2 (3) (1990), 192-217 (in Russian); English transl. in Leningrad Math. J. 2 (1991).

[8] P. L. Lions, Sur les équations de Monge-Ampère I, Manuscripta Math. 41 (1983), 1-43.

[9] N. S. Trudinger, The Dirichlet problem for the prescribed curvature equations, Arch. Rational Mech. Anal. 111 (1990), 153-179.

[10] —, Isoperimetric inequalities for quermassintegrals, preprint CMA-MR11-93. 